\title{
Les méthodes d'alimentation en eau des voies navigables Optimisation d'un projet avec écluses Les stations de pompage
}

\author{
Water supply methods for navigable waterways \\ Optimization of a project with locks \\ Pump stations
}

\author{
MM. Pommier et A. Terrier \\ Compagnie Nationale du Rhône
}

\author{
MM. Le Grand et Olivier \\ Bergeron S. A.
}

\section{Généralités}

Pour les voies navigables reliant 2 bassins, les difficultés en ce qui concerne l'alimentation en eau, intéressent principalement le bief de partage. Autrefois, ce problème était résolu par récupération des eaux disponibles au niveau de ce bief avec constitution de réserves contrôlées par un barrage.

Présentement, étant donné l'augmentation des dimensions des écluses en plan et hauteur de chute, l'utilisation de barrages réservoirs ne suffit, en principe, plus. Il faut donc faire appel à d'autres techniques.

Un calcul d'optimisation réalisé dans le cadre des études de la liaison à grand gabarit Saône-Rhin, a permis de mettre en évidence les limites d'utilisation de chacune d'entre elles.

Description de la voie navigable Saône-Rhin Bilan des ressources et des besoins en eau dans le bief de partage

Description du tronçon où se pose le problème des ressources en eau

La liaison Saône-Rhin a une longueur de $229 \mathrm{~km}$ entre la Saône et le grand canal d'Alsace. Le seuil de partage se situe à $50 \mathrm{~km}$ de celui-ci, quant au tronçon sur lequel se présentent des difficultés d'alimentation en eau, il a une longueur de $75 \mathrm{~km}$ et il comporte 11 écluses avec des hauteurs de chute maximales de 7,20 $\mathrm{m}$ sur le versant Rhône et de $24 \mathrm{~m}$ sur le versant Rhin (figure 1 ).

\section{Ressources en eau disponibles}

Elles sont constituées par les apports de 7 ruisseaux et d'une rigole dont les débits globaux sont caractérisés

en année moyenne par les valeurs ci-après:

- étiage absolu

- étiage de 10 jours

- débit semi-permanent

- débit moyen

- débit dépassé 10 jours par an

\section{Besoins en eau}

Pour assurer le fonctionnement des écluses et compenser les pertes diverses de la voie, les débits ciaprès sont nécessaires :

\section{Consommation des écluses}

Etant donné les dimensions des sas, le volume préle vé sur le bief de partage pour un cycle d'éclusage est de $73500 \mathrm{~m}^{3}$. A saturation de la voie, c'est-à-dire pour 20 cycles d'éclusage par jour, le débit permanent correspondant est de $17 \mathrm{~m}^{3} / \mathrm{s}$.

\section{Pertes d'eau}

Elles sont de trois sortes:

- les fuites aux écluses;

- les pertes par évaporation ;

- les pertes par infiltration;

et leur débit global a été estimé à :

- $220 \mathrm{l} / \mathrm{s}$ en moyenne sur l'ensemble de l'année;

$-440 \mathrm{l} / \mathrm{s}$ au maximum pendant l'été.

\section{Bilan des ressources et des besoins en eau}

Abstraction faite des pertes qui peuvent être compensées par la réserve du barrage existant de Champagney qui alimente présentement l'actuel canal Rhin-

LA HOUILLE BLANCHE/N ${ }^{\circ}$ 2/3-1981 


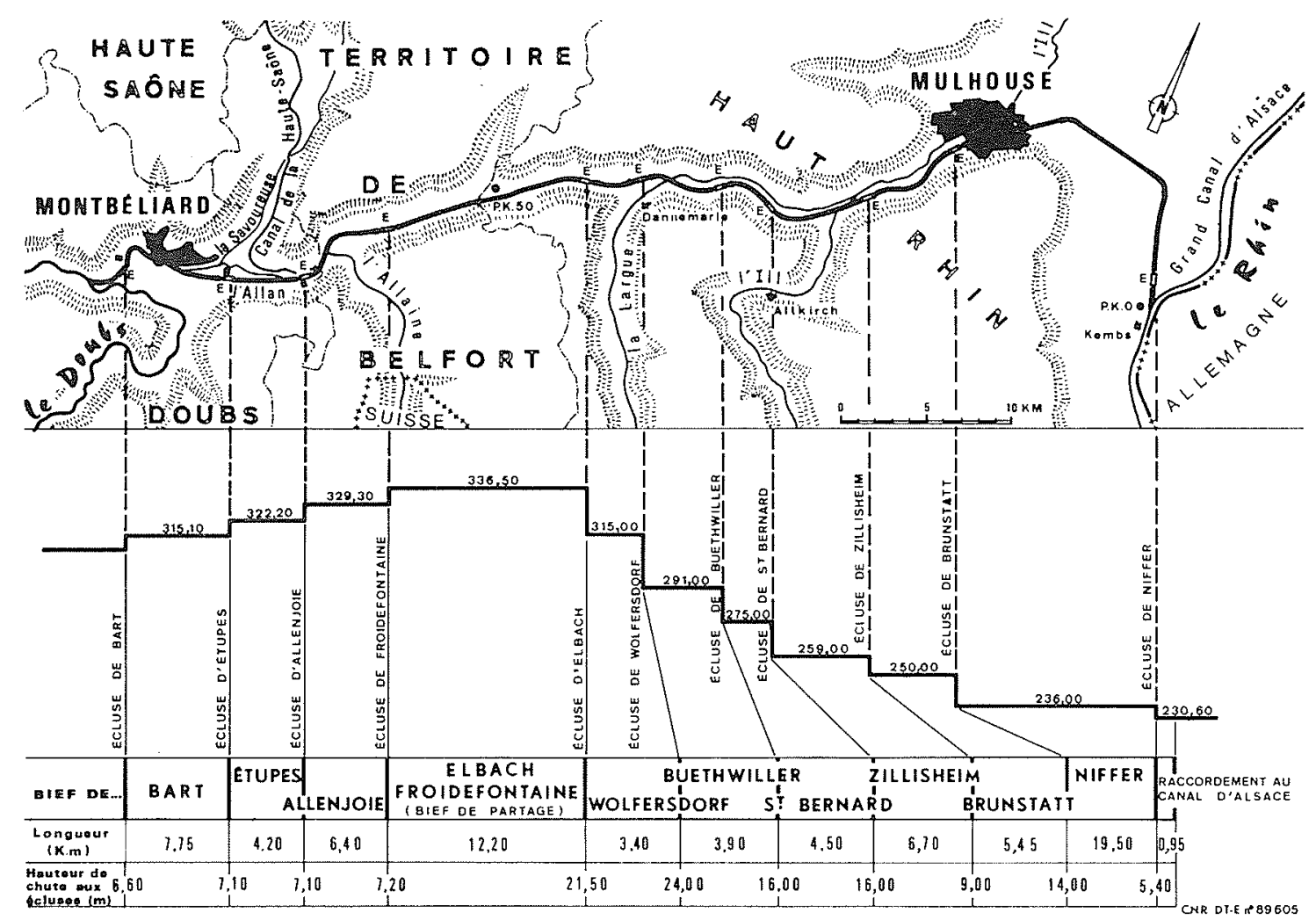

Figure 1

Rhône, les ressources en eau disponibles, très variables en cours d'année, sont en moyenne de $3,6 \mathrm{~m}^{3} / \mathrm{s}$ pour des besoins de $17 \mathrm{~m}^{3} / \mathrm{s}$.

Il est donc indispensable de faire appel à des moyens complémentaires pour assurer une exploitation normale de la voie.

\section{Moyens utilisables pour assurer l'alimentation en eau en toutes circonstances}

Trois moyens peuvent être envisagés :

- collecte et régularisation d'apports naturels au moyen d'un barrage réservoir ;

- réduction des consommations par adjonction de bassins d'épargne ;

- compensation des débits déficitaires par pompages.

Chacun de ces moyens peut à priori être utilisé seul ou en combinaison avec les autres.

\section{Barrage réservoir}

L'utilisation d'une cuvette naturelle à proximité du village de Roppe sur un ruisseau appelé Autruche et l'amenée dans cette réserve d'une partie des apports de 4 autres ruisseaux est susceptible de garantir, avec les apports naturels actuels, un débit d'étiage de $3,2 \mathrm{~m}^{3} / \mathrm{s}$ en année moyenne. En année sèche, par contre, le barrage se trouverait entièrement vide au moment le plus critique, sa prise en compte ne peut donc pas être considérée comme une possibilité de solution globale du problème d'alimentation en eau du bief de partage. Par contre son exploitation pourrait s'avérer plus économique que l'utilisation d'autres moyens complémentaires, ce qui sera examiné plus avant.

\section{Bassins d'épargne}

Ce sont des réserves annexes, en communication avec le sas de l'écluse, qui jouent le rôle d'économiseur d'eau. Le coefficient d'épargne qui caractérise cette économie est, pour des bassins de surface égale à la surface du sas de l'écluse, donné par la formule simplifiée :

$$
E=n /(n+2) \text { où } n=\text { nombre de bassins }
$$

soit :

$\begin{array}{lll}E=0,33 & \text { pour } & 1 \text { bassin } \\ E=0,5 & \text { pour } & 2 \text { bassins } \\ E=0,6 & \text { pour } & 3 \text { bassins }\end{array}$

Cette formule montre que, dans le cas étudié, les bassins d'épargne ne peuvent pas être utilisés seuls pour combler l'insuffisance des apports naturels. Par contre, ils pourront permettre de réduire les caractéristiques des stations de pompage nécessaires en tout état de cause.

\section{Stations de pompage}

$\mathrm{Au}$ vu des distances importantes existant entre le bief de partage d'une part, le Doubs et le grand canal 


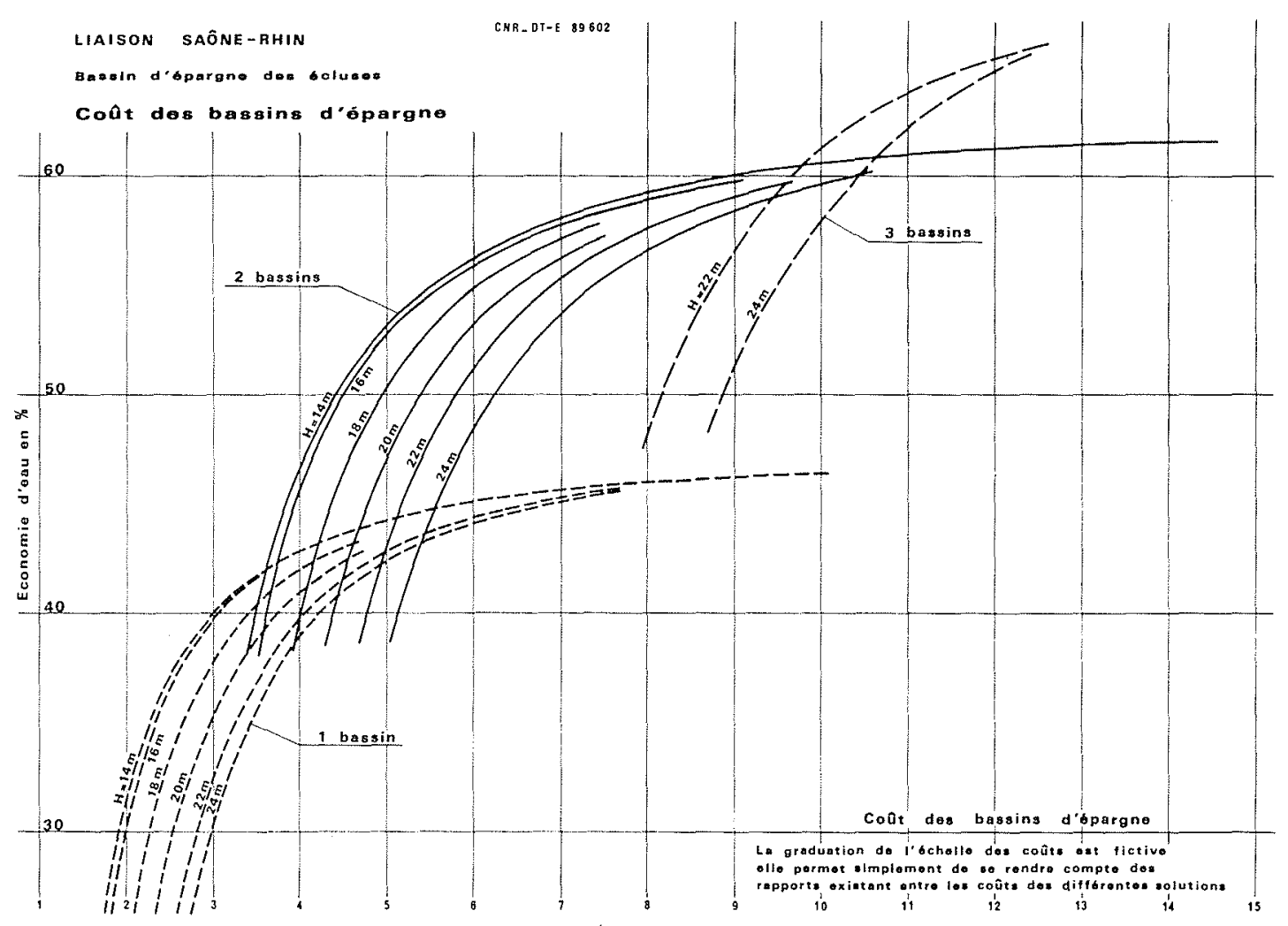

Figure 2

d'Alsace d'autre part, qui constituent les deux seules sources où l'on dispose de débits suffisants pour alimenter ces stations, il a été prévu d'implanter celles-ci au droit de chacune des écluses en utilisant le canal comme vecteur d'eau.

L'équipement de ces stations a été fixé sur la base des critères ci-après :

- débit d'apports naturels nul;

- temps maximal journalier de fonctionnement des pompes : 20 heures;

- débit nominal de la station obtenu même avec une pompe à l'arrêt ;

- pompes identiques.

Dans le cadre d'une alimentation complète par pompage, le débit unitaire des pompes est susceptible de varier de $5,25 \mathrm{~m}^{3} / \mathrm{s}$, pour un équipement à 4 pompes à $10,20 \mathrm{~m}^{3} / \mathrm{s}$, pour un équipement à 3 pompes.

Il s'agit donc de stations de pompage relativement importantes.

\section{Optimisation du projet}

Afin d'obtenir le coût total le plus faible possible, il convient de combiner les différents moyens et en premier lieu de préciser la structure des divers coûts qui entrent dans le bilan général.
Structure de coût des équipements permettant de combler l'insuffisance des apports naturels

\section{Barrage réservoir}

Les coûts d'exploitation et d'entretien pouvant être considérés comme négligeables, seul a été pris en compte le coût d'investissement.

\section{Bassins d'épargne}

Leurs coûts sont donnés par le graphique $n^{\circ} 2$. Ils ont été établis sur la base de bassins d'épargne en béton et de forme carrée, de manière à réduire le génie civil, en outre, les dépenses d'exploitation et d'entretien ont été considérées comme négligeables.

Ces coûts varient en fonction des coefficients d'épargne et des hauteurs de chutes des écluses auxquelles ils sont accolés, par ailleurs, à partir d'un certain seuil du coefficient d'épargne, il y a intérêt à modifier le nombre des bassins.

D'autre part, si l'on rapporte ces coûts au volume épargné à chaque cycle, (voir graphique $\mathrm{n}^{\circ} 3$ ) on constate que celui-ci passe par un minimum.

Tenu compte du fait que l'adjonction d'un bassin d'épargne permet de réduire les coûts de pompage, il peut donc exister une valeur du coefficient d'épargne à partir de laquelle les économies réalisées sur les dépenses de pompage ne compensent plus les surcoûts d'investissement résultant de l'accroissement de l'épargne.

L'objectif du calcul d'optimisation sera de déterminer cette limite si elle existe. 


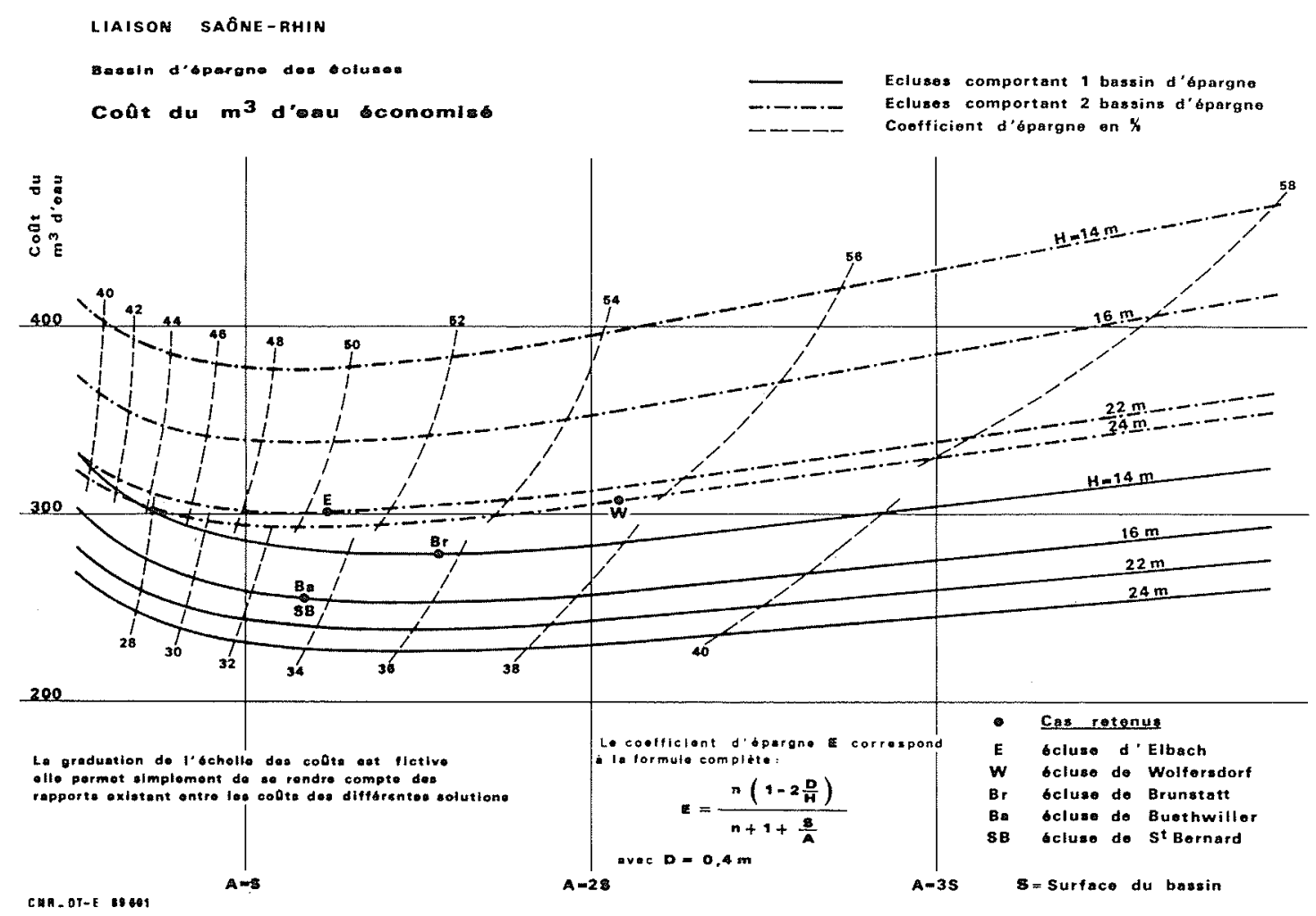

Figure 3

\section{Station de pompage}

Pour ce type d'ouvrage on doit considérer à la fois les coûts d'investissement et les coûts de l'énergie consommée. Ces deux composantes de prix se réduisent évidemment avec la consommation d'eau effective à l'écluse correspondante.

Un autre moyen de jouer sur le coût des pompages, pour une consommation donnée à l'écluse, consiste à faire varier le nombre de pompes installées dans la station. Mais ceci se traduit par des effets différents sur les diverses composantes du coût de pompage. En effet, si on réduit le nombre total des pompes:

- le coût d'investissement de la station diminue, car l'accroissement du prix unitaire des pompes reste inférieur à l'économie réalisée en réduisant le nombre total de pompes ;

- la redevance de puissance souscrite augmente, car la condition de pouvoir assurer le passage du trafic avec une pompe à l'arrêt entraîne l'installation d'une surcapacité de pompage qui augmente lorsque le nombre de pompes diminue ;

- le coût de l'énergie consommée diminue, car l'accroissement de la surcapacité de pompage permet de bénéficier au mieux des tarifs d'heures creuses.

On cherchera donc tout d'abord à combiner les stations de pompage avec un moyen permettant de réduire le déficit en eau aux diverses écluses, puis on ajustera le nombre de pompes à chaque station afin d'aboutir au coût global minimum.

\section{Critère d'implantation des bassins d'épargne}

On démontre qu'en première approximation, le gain obtenu sur le coût de pompage, par l'adjonction d'un bassin d'épargne, est proportionnel à la valeur du produit $H^{2} \times \Delta$ où :

- $H$ est la hauteur de chute à l'écluse considérée; $-\Delta$ est la réduction du coefficient de consommation $(C C=1-E)$ qui résulte de l'implantation du nouveau bassin par rapport à la situation antérieure.

Le critère d'implantation cherché consiste donc à dresser pour les diverses écluses concernées le tableau des valeurs de $H^{2} \times \Delta$ correspondant aux diverses hypothèses d'épargne. Partant d'une solution de base sans épargne, il suffit alors d'installer les bassins successifs en suivant l'ordre des valeurs décroissantes du produit $H^{2} \times \Delta$, ce qui garantit que chaque nouveau bassin se trouve bien placé à l'endroit où il procurera le gain maximum sur les coûts de pompage.

\section{Programme de calcul des coûts capitalisés de pompage}

La définition du coût global d'une solution avec stations de pompage nécessite la prise en compte des coûts capitalisés de pompage.

Or la définition des coûts quotidiens de pompage va dépendre de plusieurs parametres : apports naturels dans les différents biefs, niveau de trafic, prix de l'énergie suivant les horaires et les saisons de fonctionnement. Et l'actualisation de ces couts dépendra à son tour des hypothèses faites sur la loi d'évolution du trafic dans le temps et sur le tatix d'actualisation à utiliser. 
Compte tenu du nombre des paramètres à prendre en compte, on a établi un programme de calcul permettant d'obtenir le coût global capitalisé des pompages pour chaque solution envisagée.

L'addition du résultat foumi par ce programme et des coûts d'investissement définis par ailleurs permet d'obtenir le coût global de chaque solution et donc d'aborder la phase de l'optimisation proprement dite.

\section{Optimisation du projet combinant des bassins d'épargne ct des stations de pompage}

On part d'une solution de base ne comportant que des stations de pompage (on a vu plus haut que c'est le seul moyen qui permette, à lui seul, d'assurer le fonctionnement de la voie d'eau). Et on chiffre le coût global de cette solution en ajoutant le coût actualisé des pompages aux coûts d'investissements.

On utilise alors le critère d'implantation des bassins d'épargne afin d'accroître progressivement le nombre de bassins dans l'ordre de leur intérêt économique décroissant. Et on calcule le coût global de chacune des solutions ainsi définies, afin de permettre la comparaison avec la solution de base.

Dans le cas de la liaison Saône-Rhin, l'étude a été faite pour :

- deux lois d'accroissement du trafic, à partir d'un trafic initial de 5 cycles par jour:

. accroissement de 0,5 cycle/jour par an;

. accroissement de 1 cycle/jour par an;

- trois taux d'actualisation: 5,7 et $10 \%$.

Le graphique $n^{\circ} 4$ donne l'allure de l'évolution des prix des diverses solutions par rapport au prix de la solution de base.

Ce graphique permet de constater qu'il existe bien toujours un nombre optimum de bassins d'épargne et que cet optimum est :

- pratiquement indépendant de la loi d'évolution du trafic ;

- fortement dépendant de la valeur du taux d'actualisation.

Etant donné l'évolution prévisible des coûts de l'énergie et compte tenu de la longue durée de vie d'un tel
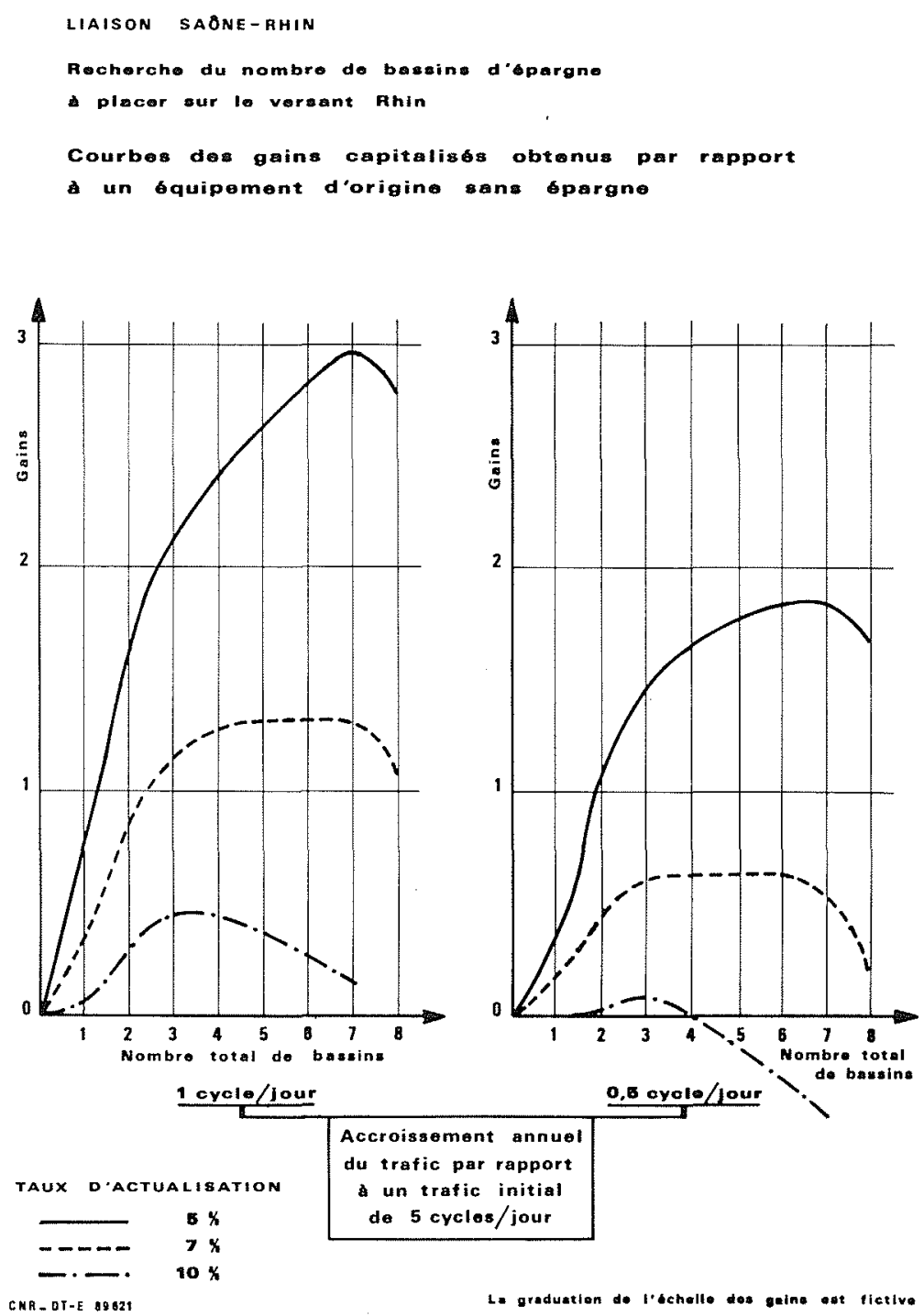

Figure 4 
aménagement, il a été admis un taux d'actualisation relativement faible, compris entre 5 et $7 \%$. On arrive ainsi à un nombre total de sept bassins d'épargne.

L'étude ayant été conduite avec des bassins de surface égale à celle des sas, on constate alors que les volumes consommés à chaque cycle par certaines écluses sont assez proches les uns des autres. On a donc ajusté la surface des bassins afin d'obtenir des volumes consommés identiques, ce qui permet de réduire les problèmes de marnage dans les biefs, les débits de pompage installés étant identiques. Et finalement le projet définitif correspond aux valeurs des coefficients d'épargne indiqués sur le graphique $n^{\circ} 3$.

\section{Intérêt économique d'un barrage réservoir}

Le barrage de Roppe étant vide en année sèche ne permet pas de réduire les puissances de pompage installées, par contre l'étiage de $3,2 \mathrm{~m}^{3} / \mathrm{s}$ qu'il permet de garantir en année moyenne peut se traduire par un gain sur les coûts de pompage.

Le calcul des coûts capitalisés de pompage a donc été refait dans cette hypothèse, mais il montre que le gain obtenu n'est que de 20 à $45 \%$ du coût du réservoir suivant les hypothèses d'accroissement de trafic et de taux d'actualisation. L'adjonction d'un tel réservoir à l'équipement avec bassins d'épargne et stations de pompage n'est donc pas économiquement justifiée.

Fquipement et commande des stations de pompuge

1. surete doxploitation de la vole d'eau, le cout te lonerge of lo cout dentretien d'un parc de matérels importants aneneront à rechercher des types de pompes flables, ayant un bon rendement et nécessi- tant des coûts et des fréquences d'intervention aussi faibles que possible. Le matériel le mieux adapté à ce point de vue semble être constitué par les pompes du type à volute avec montage du moteur directement sur les pompes, sans plancher intermédiaire.

Les précautions habituelles devront bien entendu être prises au niveau de la conception des stations afin de minimiser les pertes de charges, quel que soit le nombre de pompes en service, et en tenant compte des problèmes d'encrassement des prises d'eau par les matériaux flottants ou le gel.

On peut noter enfin que l'existence des bassins d'épargne et les ajustements faits sur leurs surfaces pour ramener les volumes consommés par des écluses voisines à des valeurs identiques, sont des éléments simplificateurs importants pour la conception du système de commande des stations de pompage.

\section{Conclusion}

Des éléments exposés on peut retenir que :

- l'alimentation naturelle du bief de partage d'une voie navigable constituée par une chaîne d'écluses à grand gabarit ne peut pas assurer le fonctionnement gravitaire complet de la voie ;

- un barrage réservoir ne peut se justifier économiquement que si sa capacité et ses conditions de remplissage permettent de réduire suffisamment les stations de pompage nécessaires par ailleurs ;

- le franchissement des seuils par des chaînes d'écluses a grand gabarit remet en valeur l'intérêt des bassins d'épargne ;

- la combinaison d'un ensemble de bassins d'épargne et de stations de pompage peut se faire de façon à minimiser le coût total capitalisé du projet et la méthode exposée peut être appliquée à tout projet du même type. 